\title{
Determination of the Analytical Relationship between Refractive Index and Density of $\mathrm{SiO}_{2}$ Layers
}

\author{
W. RzODKIEWICZ* AND A. PANAS \\ Institute of Electron Technology, al. Lotników 32/46, 02-668 Warsaw, Poland
}

\begin{abstract}
The main goal of the work was the elaboration of the analytical functional relationship between refractive index $n$ and density $\rho$ of $\mathrm{SiO}_{2}$ layers on silicon substrates. Such $\rho(n)$ relationship will give possibility to determine elastic and non-elastic strains in $\mathrm{SiO}_{2}$ layers on silicon substrates. Ellipsometric measurements by using variable angle spectroscopic ellipsometer of J.A. Woollam Company allowed determination of thicknesses and refractive indexes of silica layers. Measured $\mathrm{SiO}_{2}$ masses and calculated volumes of the layers gave possibility to define the degree of densification of silicon dioxide layers on silicon substrates. The Hill approximation function curve turned out to be the best fitting. The obtained Hill curve shows saturation for the density of silicon dioxide equal to $c a .4 .53 \mathrm{~g} / \mathrm{cm}^{3}$. This value corresponds to the value nearby the one of the crystalline polytypic silicon dioxide (stishovite). It seems to be physically established that degree of densification tends to the limiting value.
\end{abstract}

PACS numbers: 81.40.Tv, 61.82.Ms

\section{Introduction}

Managing internal mechanical stress is an essential issue to provide very good performance and high reliability in advanced CMOS manufacturing. Strain can be generated in MOS devices at many technological steps. Due to scaling down of integrated circuits and rising up functionality in the modern electronics technology, good understanding of the compaction (connected with the elastic strains) and decompaction (connected with permanently irreversible strains) mechanisms in the $\mathrm{Si}-\mathrm{SiO}_{2}$ system is necessary. Additionally, when the structure dimension is going down, their mechanical and optical properties may differ from the corresponding values of the bulk medium. Thus, studies of both mechanical and optical properties are very essential.

In connection with this, the main goal of our work was to elaborate the functional relationship between the refractive index $n$ and the density $\rho$ (as the degree of densification) of silicon dioxide layers on silicon substrates.

\section{Experimental details}

Czochralski-grown, $n$-type both $\langle 100\rangle$ and $\langle 111\rangle$ oriented silicon substrates have been applied in our investigations. We started with double-side polished 4 inch wafers. Subsequently, the wafer were subjected to thermal oxidation process at $1000{ }^{\circ} \mathrm{C}$ in either dry oxygen (in order to grow silicon dioxide layers on $\langle 100\rangle$ silicon substrates with the thickness of approximately 10, 15, 20, 25, $30,35,40,45,50 \mathrm{~nm}$ ) and water vapour (in order to grow oxide layers on $\langle 111\rangle$ silicon substrates with the thickness

\footnotetext{
* corresponding author; e-mail: rzodki@ite.waw.pl
}

of approximately 50,100,150, $200 \mathrm{~nm}$ ). The thickness of $\mathrm{SiO}_{2}$ layers and their refractive indexes were determined using a variable angle spectroscopic ellipsometer (VASE) with rotated analyser of J.A. Woollam Inc. Co. But, before thermal oxidation, the Fizeau interferometer was applied for the investigation of the quality and shape of silicon substrates surface.

\section{Optical model}

By spectroscopic ellipsometry we get spectral characteristics of $\Psi$ (ratio of amplitudes after reflection for polarizations $p$ and $s$ ) and $\Delta$ (phase change after reflection for polarizations $p$ and $s$ ). To extract quantitative information about a sample, in most cases, it is necessary to construct an investigated structure model consisting of suitable number of layers of specified thicknesses. Then we can fit calculated and experimental $\Psi$ and $\Delta$ data to compare generated and measured data. It is known that for the sake of simplicity and convenience, the Cauchy dispersive model is usually being used to fit ellipsometric data for dielectrics and semiconductors in spectral regions where they are transparent (i.e. where the extinction coefficient is zero). The Cauchy model allows determination of optical coefficients (complex refractive index) and thickness of analyzed layers of given structure. The relationship, which allows determination of the $\mathrm{SiO}_{2}$ refractive index by Cauchy model, is given by the Cauchy dispersion formula [1]:

$$
n(\lambda)=A+\frac{B}{\lambda^{2}}+\frac{C}{\lambda^{4}}+\ldots,
$$

where $A, B, C$ are calculated fitting coefficients, and $\lambda$ stands for wavelength in $\mu \mathrm{m}$.

The spectroscopic ellipsometry analysis for all the investigated spectral range was performed. The Sellmeier 
model which is also being applied to fit experimental and generated data for dielectrics in spectral regions where they are not absorbing has not been considered. However, when the spectral range extends significantly beyond the visible into the mid-infrared, the refractive index of many transparent materials (such as among other things the silicon dioxide) trend downward. Then, the Sellmeier model is strongly recommended for the above mentioned spectral range (mid-infrared range) [2].

The optical model which was used for ellipsometric data analyses consisted of a silicon dioxide layer as a Cauchy layer and a silicon substrate (fixed data taken from [3]). In the applied model, the two parameters of Cauchy $(A, B)(1)$ and $\mathrm{SiO}_{2}$ layer thickness were fitted (the parameter $C$ was fixed as 0 ). In opposition to what has been presented in papers [4-6], the interface layer between silicon substrate and silicon dioxide was not considered.

\section{Results and discussion}

For the sake of the quality and shape of silicon substrates surface, before thermal oxidation, all substrate wafers were undergone interferometric measurements by means of the Fizeau interferometer (the light source was the laser diode operating at $635 \mathrm{~nm}$ ).

Analysis of the mass, the volume and the density errors have been performed by the complete differential method [7]. In this analysis, the significant changes in density of $\mathrm{SiO}_{2}$ layer (of the order of $0.1-0.3 \mathrm{~g} / \mathrm{cm}^{3}$ ), at least $0.2 \mathrm{mg}$ error of the mass, were detected. Thus, the accuracy of the oxide mass measurement played the most important role at the determination of $\mathrm{SiO}_{2}$ layer mass. On the basis of this analysis we affirmed that the balance with accuracy at least $0.01 \mathrm{mg}$ should be used. Therefore, in our investigations such balance was applied.

Once the mass of an $\mathrm{SiO}_{2}$ layer is measured and its volume is known (calculated as the product of the oxide thickness and surface area of the layer), one may determine directly the density of the layer.

Next, the density of $\mathrm{SiO}_{2}$ layers obtained in this way were compared with that determined from the value of the refractive index (established in the course of ellipsometric measurements). The Lorentz-Lorenz formula [8-10] ( $\mathrm{L}-\mathrm{L})$ is one of the common used equation relating oxide density to its refractive index

$$
\Pi=\frac{1}{3} N_{\mathrm{A}} \alpha=\frac{n^{2}-1}{n^{2}+2} \frac{M}{\rho},
$$

where $\Pi$ - molar polarization, $N_{\mathrm{A}}$ - Avogadro's number, $\alpha$ - mean polarizability, $M-$ molecular mass, $\rho$ - density. Substituting the following values: $M=$ $60.08 \mathrm{~g} / \mathrm{mol}$ (molecular mass of $\mathrm{SiO}_{2}$ ), $\rho=2.2 \mathrm{~g} / \mathrm{cm}^{3}$ and $n=1.46$ (density and refractive index of the relaxed $\mathrm{SiO}_{2}$, respectively), we obtain molar polarization for $\mathrm{SiO}_{2}$ layer $\Pi=7.4797 \mathrm{~cm}^{3} / \mathrm{mol}$ and (1) becomes

$$
\rho=8.0324 \frac{n^{2}-1}{n^{2}+2} .
$$

Other formulae relating oxide density and its refractive index are: the Gladstone-Dale equation [10, 11] (G-D):

$$
\rho=4.785 n-4.784,
$$

the empirical $\rho(n)$ relationship for $\mathrm{SiO}_{2}$ layer derived by Taniguchi et al. [12] (Ta):

$$
\rho=7.81(n-1)^{1.63}
$$

and the Drude equation $[10,13]$ :

$$
\rho=1.944\left(n^{2}-1\right) \text {. }
$$

The last formula has usually been applied for study of the optical properties of various liquids (such as chlorobenzene, methanol, water, etc.).

Dependence of silicon dioxide density, $\rho_{o x}$ on the oxide refractive index $n$ for back-side oxide layer against a background of data given in the literature is shown in Fig. 1.

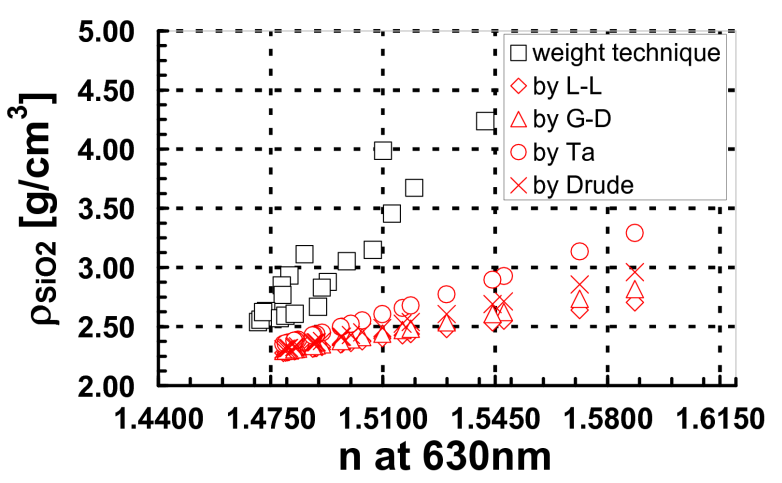

Fig. 1. Dependence of silicon dioxide density, $\rho_{\mathrm{SiO}_{2}}$ (experimentally determined by weight technique) on the oxide refractive index, $n$ (determined by spectroscopic ellipsometry).

The very good evidence for the correctness of the obtained experimental data points is dependence of silicon dioxide density, $\rho_{\mathrm{SiO}_{2}}$ on the oxide thickness, $t_{\mathrm{ox}}$ (determined on the assumed optical model) illustrated in Fig. 2. Such regularity results from the observation of two trends of the presented curve. The first, linear dependence is detected for the oxide thicknesses greater than $50 \mathrm{~nm}$. But, as the oxides thinner than $50 \mathrm{~nm}$ are concerned (closer to the $\mathrm{Si}-\mathrm{SiO}_{2}$ interface), the power dependence $\rho\left(t_{\text {ox }}\right)$ is detected.

On the basis of the density values obtained for the layers during all so far done weight experiments [14] curve fitting analysis was performed. It was necessary for finding of the corresponding curve giving proper relationship between the oxide density and its refractive index. The best curve of several investigated curves (inter alia the logarithmic function with three parameters and Langmuir approximation) turned out to be the Hill function (Fig. 3).

Dashed and dotted lines in Fig. 3 are confidence and prediction bands (for the $95 \%$ confidence level), respectively.

The $\rho(n)$ relationship using the Hill approximation function is given by 


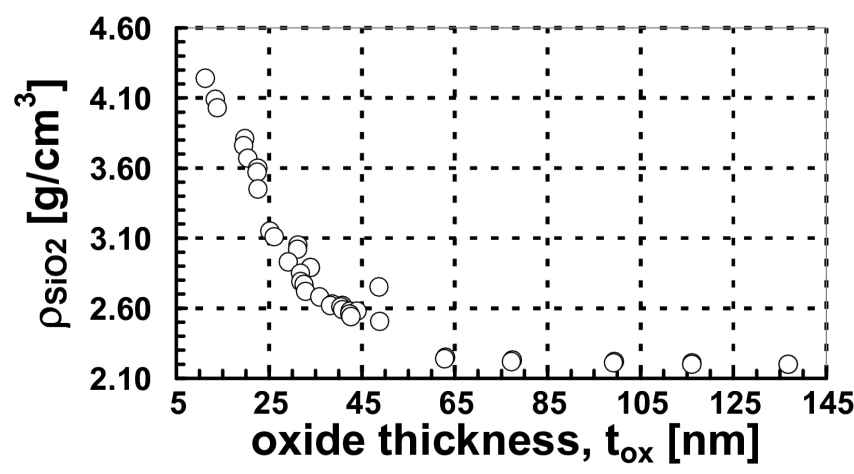

Fig. 2. Dependence of silicon dioxide density, $\rho_{\mathrm{SiO}_{2}}$ (experimentally determined by weight technique) on the oxide thickness, $t_{\mathrm{ox}}$ (determined by spectroscopic ellipsometry) on $n\langle 100\rangle$ Si substrate.

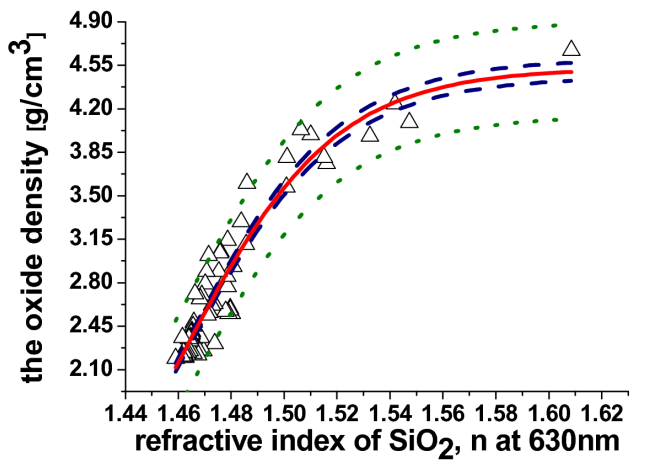

Fig. 3. Dependence of silicon dioxide density, $\rho_{\mathrm{SiO}_{2}}$ (experimentally determined by weight technique) on the oxide refractive index, $n$ (determined by spectroscopic ellipsometry) on $n\langle 100\rangle$ Si substrate for both front-side and back-side layers as well as the best fitting curve (the Hill approximation function) with $95 \%$ confidence and prediction bands.

$$
\rho=4.531 \frac{n^{51.818}}{n^{51.818}+1.4627^{51.818}} .
$$

The above $\rho(n)$ relationship indicates on the saturation for the density of the oxide at the value $c a .4 .53 \mathrm{~g} / \mathrm{cm}^{3}$. Such obtained value of density resembles the value of density characteristic of crystalline polymorph of silica (stishovite) given in the literature for example as $c a .4 .35$ $[15] \mathrm{g} / \mathrm{cm}^{3}$. It seems to be physically explained that degree of densification tends to the limiting value.

\section{Conclusions}

In this work, we have elaborated the new analytical functional relationship between refractive index $n$ and density $\rho$ of $\mathrm{SiO}_{2}$ layers on silicon substrates. The Hill function curve with three parameters turned out to be the best fitting curve corresponding to the experimental data determined by weight technique (Fig. 3).

\section{References}

[1] Software for VASE and M-44 ellipsometers - Guide to Using WVAS 32, J.A. Woollam Co. Inc., 1991.

[2] T. Tiwald, http://www. jawoollam.com/ toolbox.html, 2009, Newsletters Articles 10 Sellmeier Dispersion.

[3] J.A. Woollam Co. Inc. WVASE 32 program v. 3.441, tabulated at UNL, Lincoln University, Nebrasca, USA, 1991.

[4] D.E. Aspnes, J.B. Theetn, Phys. Rev. Lett. 43, 1046 (1979).

[5] C.M. Herzinger, B. Johs, W.A. McGahan, J.A. Woollam, W. Paulson, J. Appl. Phys. 83, 3323 (1998).

[6] C. Zhao, P.R. Lefebvre, E.A. Irene, Thin Solid Films 313-314, 286 (1998).

[7] J.M. Thomas, Mathematics 22, 109 (1936).

[8] L. Banyai, P. Gartner, Phys. Rev. B 29, 728 (1984).

[9] K.E. Oughstun, N.A. Cartwright, Opt. Express 11, 1541 (2003).

[10] K. Vedam, P. Limsuwan, J. Chem. Phys. 69, 4772 (1978).

[11] W.A. Pliskin, J. Vac. Sci. Technol. 14, 1064 (1977).

[12] K. Taniguchi, M. Tanaka, C. Hamaguchi, J. Appl. Phys. 67, 2195 (1990).

[13] P. Drude, Lehrbuch der Optik, Lepzig 1900.

[14] W. Rzodkiewicz, A. Panas, J. Telecommun. Inf. Technol. 3, 44 (2007)

[15] Sheng-Nian Luo, Lianqing Zheng, O. Tschauner, J. Phys., Condens. Matter. 18, 659 (2006). 